\title{
Structural and Spectral Characteristics of the Cross-linked Dimer Derived from Electrooxidation of Cyclic 1, $N^{2}$-Propanoguanosine
}

\author{
Hiroya MuraKami, Yukihiro EsaKa, and Bunji UNO ${ }^{\dagger}$ \\ Gifu Pharmaceutical University, 1-25-4 Daigaku-nishi, Gifu 501-1196, Japan
}

\begin{abstract}
The acetaldehyde-derived cyclic propano adduct of $2^{\prime}$-deoxyguanosine was easily oxidized electrochemically into the cross-linked dimer as an oxidative product. The structural and spectroscopic characteristics of the dimer were investigated by MS, ${ }^{1} \mathrm{H}$ and ${ }^{13} \mathrm{C}-\mathrm{NMR}, \mathrm{UV}$, and DFT calculations. The dimer formation was inferred from a molecular ionic peak of $m / z, 705\left([(2 M-2 \mathrm{H})+\mathrm{H}]^{+}, M\right.$ being the molecular weight of the monomer) on the ESI-MS spectra and the chemical formula as $\mathrm{C}_{28} \mathrm{H}_{36} \mathrm{~N}_{10} \mathrm{O}_{12}$ provided by the high-resolution ESI-MS results. The C2-N5 linkage between the two monomers in the dimer was deduced from the ${ }^{1} \mathrm{H}$ - and ${ }^{13} \mathrm{C}-\mathrm{NMR}$ spectral results. In addition, the correlations in the 2-dimensional NMR spectra (DQF-COSY and HMBC) were consistently explained by the structure of the C2-N5 cross-linked dimer. UV spectral measurements also support the C2-N5 linking in the dimer formation. The formation of the cross-link dimer as an oxidative lesion of the acetaldehyde-derived cyclic propano adduct of guanosine is expected to interfere with DNA replication and to contribute to acetaldehyde-mediated genotoxicity.
\end{abstract}

(Received December 6, 2010; Accepted January 12, 2011; Published March 10, 2011)

\section{Introduction}

It is well known that oxdative damage to the bases in DNA is the origin of alterations of the genetic code that lead to mutations and increase the predisposition to several diseases such as cancer. A recent and exciting prospect in the area of the chemistry of oxidative DNA damage lies in understanding the mechanism for formation of interstrand and intrastrand cross-links, which are expected to interfere with DNA replication and to contribute to genotoxicity. ${ }^{1,2}$ Our recent attention has been directed to the acetaldehyde-derived cyclic $1, N^{2}$-propanodeoxyguanosine adduct (CPrG), 3-(2'-deoxyribos-1'-yl)-5,6,7,8tetrahydro-8-hydroxy-6-methylpyrimido[1,2-a]purine-10(3H) one, converted from 2 -deoxyguanosine $(\mathrm{G})$ in terms of genotoxic, mutagenic and carcinogenic effects of aldehydes arising from exogenous and endogenous sources such as smoking and drinking. ${ }^{3-5}$ Derivation of CPrG has frequently occurred in calf thymus DNA and cultured HL-60 cells during exposure to acetaldehyde $^{6}$ and in human DNA from lung and liver, ${ }^{7}$ and the cross-link formation of CPrG with $\mathrm{G}$ in duplex DNA has been detected as already documented in the literature, ${ }^{8,9}$ suggesting its involvement in mutagenesis and carcinogenesis. On the other hand, it is well recognized that CPrG is very sensitive during a single-electron oxidation and easily causes oxidative degradations of the ring-opened propano adduct and its ring-opening equivalents. ${ }^{3}$ The formation of the oxidative degradation products, however, cannot be explained by considering the cross-link formation as the point-mutations by acetaldehyde, because they induce absolute blocking of DNA replication.

Recently, we have reported that the electrochemical

† To whom correspondence should be addressed.

E-mail: uno@gifu-pu.ac.jp electron-drawing of calf thymus DNA containing the acetaldehyde-derived CPrG results in dimerization of vicinal CPrG's. ${ }^{10}$ The formation of the dimer was analyzed with a LC/ESI-MS detector set in a selected ion monitoring mode of $\mathrm{m} / \mathrm{z} 441$ corresponding to the molecular ion of the dimer of the cyclic propano adduct of guanine after depurination with hydrochloric acid. This strongly suggests that oxidation damage of DNA exposed to acetaldehyde induces the cross-link dimer (D-CPrG) formation of CPrG in double-stranded DNA. It is well recognized that electron-loss center in DNA ultimately ends up at guanine residues via hole migration through a $\pi$-stacking of the DNA duplex. ${ }^{11,12}$ Likewise, oxidation stress in duplex DNA is localized at a low oxidation potential site of the CPrG-CPrG step acting as a trap in long-range hole migration through the DNA duplex; the resulting D-CPrG is formed at the vicinal G-G base sequence. However, the real structure of D-CPrG remains dubious. In this work, D-CPrG was electrosynthesized from CPrG and the structural and spectral characteristics were investigated at a nucleoside level by NMR, UV, MS and molecular orbital calculations.

\section{Experimental}

\section{Chemicals}

G was purchased from Tokyo Chemical Industry Co., Ltd. (Japan). CPrG was synthesized as reported previously. ${ }^{13}$ Crotonaldehyde and $\mathrm{L}(+)$-arginine used for synthesis of CPrG were purchased from Kishida Chemical Co., Ltd. (Japan). The other reagents were purchased from Nacalai Tesque (Japan). These reagents were used as received without further purification.

Controlled potential electrolysis of CPrG was performed with a three electrode system consisting of a carbon-felt working electrode, a coiled platinum counter electrode, and an $\mathrm{Ag} / \mathrm{AgCl}$ 


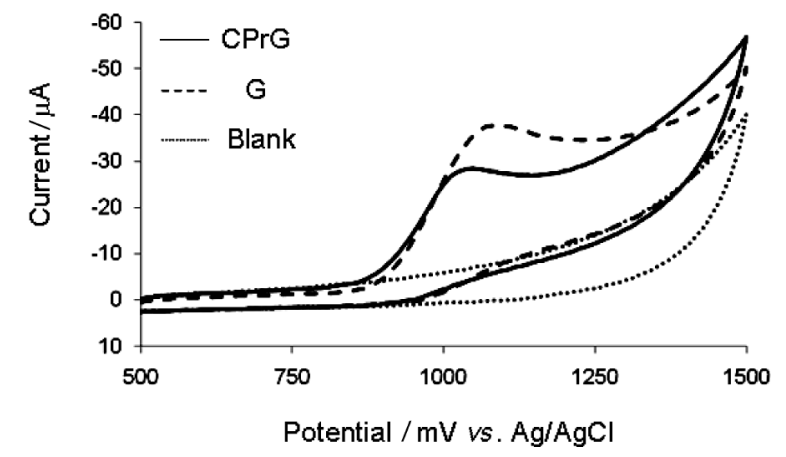

Fig. 1 Cyclic voltammograms of $1 \mathrm{mmol} \mathrm{dm}^{-3} \mathrm{G}$ and $1 \mathrm{mmol} \mathrm{dm}^{-3}$ $\mathrm{CPrG}$ in phosphate buffer, recorded with a $\mathrm{GC}$ electrode at a scan rate of $100 \mathrm{mV} \mathrm{s}^{-1}$.

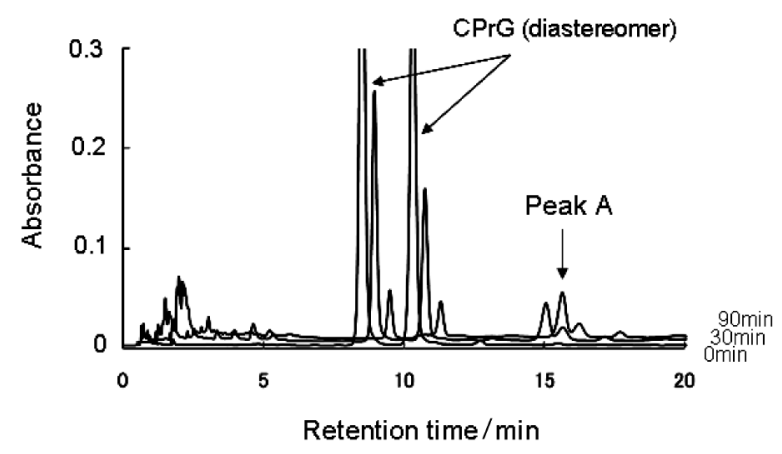

Fig. 2 HPLC elution profile for the electrolyzed solution of CPrG. HPLC separation was performed on an ODS column with a mobile phase consisting of water and methyl alcohol (linear gradient: 10 - 23\% methyl alcohol over $20 \mathrm{~min}$ ) at a flow rate of $0.3 \mathrm{~mL} \mathrm{~min}^{-1}$.
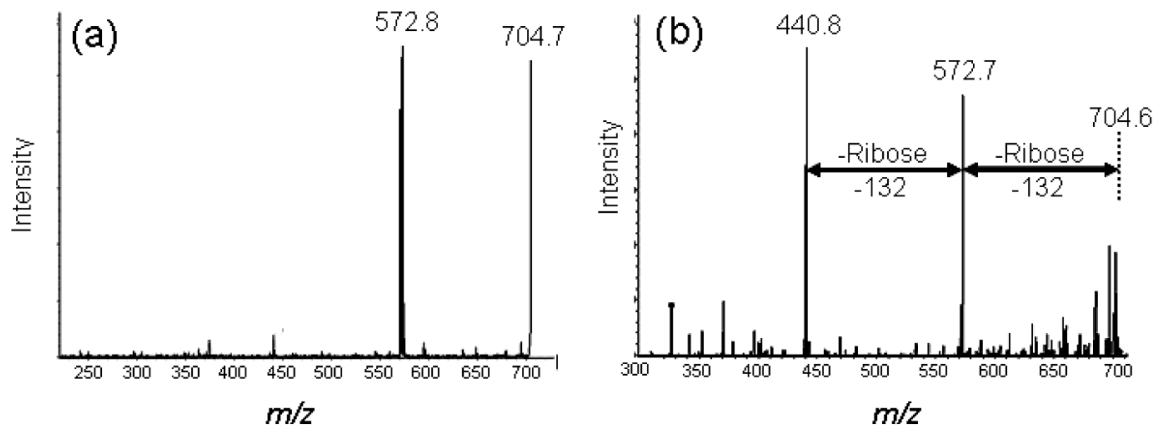

Fig. 3 (a) ESI-MS spectra of the fraction corresponding to Peak A in Fig. 2 and (b) MS/MS spectra of the molecular peak $(\mathrm{m} / \mathrm{z}=705)$ in $(\mathrm{a})$.

reference electrode. The electrooxidation product was isolated from the reaction mixture by a reversed-phase $\mathrm{C}_{18}$ Cosmosil packed column ( $5 \mathrm{C}_{18}$-MS, Nakalai Tesque, Japan) with isocratic elution by $10 \%$ methyl alcohol in water at a flow-rate of

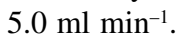

\section{$M S$, electrochemical and spectral measurements}

MS/MS analysis was performed on an Esquire 3000+ (Brucker, Germany). High-resolution MS analysis was performed on a JMS-T100LP (JEOL, Japan). Cyclic voltammetry was performed with a three-electrode system consisting of a glassy carbon working electrode, a coiled platinum counter electrode, and an $\mathrm{Ag} / \mathrm{AgCl}$ reference electrode. The voltammograms were recorded with a BAS-50W electrochemical workstation. UV spectra were measured in the usual manner at room temperature with a Shimadzu spectrophotometer (Multispec 1500). NMR spectra were observed in DMSO- $d_{6}$ and DMSO- $d_{6}+\mathrm{D}_{2} \mathrm{O}$ with JEOL NMR spectrophotometers (Model JNM-EX400 and JNM-AL400).

\section{MO calculations}

DFT calculations (B3LYP) with 6-31+G(d,p) basis sets were carried out to get the optimized geometries of CPrG and D-CPrG using the Gaussian 03 program (Revision E.01). ${ }^{14}$ To interpret the experimental absorption spectra, CNDO/S-CI calculations were carried out. The parameters necessary for the calculations were taken from the literature of Jaffé's group and others. ${ }^{15-20}$ Tow-center repulsion integrals were evaluated using the Nishimoto-Mataga equation. ${ }^{21}$ Only the one-electron transition was taken into account for $\mathrm{CI}$ calculations. Optimized molecular geometries obtained by the B3LYP/6-31+G(d,p) calculations were used for the calculations.

\section{Results and Discussion}

Electrooxidative product of acetaldehyde-derived $\mathrm{CPrG}$ and the MS spectra

It is well recognized that $\mathrm{CPrG}$ is susceptible to oxidation; however, the oxidation potential is not clear. Figure 1 shows the cyclic voltammograms of $\mathrm{G}$ and $\mathrm{CPrG}$. The voltammograms showed an irreversible step, but we can find that $\mathrm{CPrG}\left(E_{\text {ap }}=\right.$ $1.04 \mathrm{~V}$ vs. $\mathrm{Ag} / \mathrm{AgCl}$ ) is more easily oxidized compared to $\mathrm{G}$ $\left(E_{\text {ap }}=1.08 \mathrm{~V} v s . \mathrm{Ag} / \mathrm{AgCl}\right)$. The B3LYP/6-31+G(d,p) calculation results also support the susceptibility to oxidation for $\mathrm{CPrG}$, showing that the calculated ionization potentials (Koompan's theorem) are 5.965 and $5.760 \mathrm{eV}$ for $\mathrm{G}$ and $\mathrm{CPrG}$, respectively. Controlled potential electrolysis for $\mathrm{CPrG}$ was performed on a carbon felt electrode set at $1.1 \mathrm{~V} v s$. $\mathrm{Ag} / \mathrm{AgCl}$ in phosphate

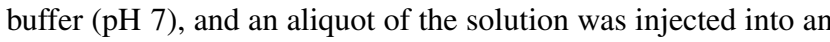
HPLC. A prominent peak corresponding to the oxidative lesion of CPrG was observed as it increased over electrolyzed time, as shown in Fig. 2. On the basis of HPLC analyses, the apparent conversion ratio of $\mathrm{CPrG}$ into the product at the stationary state attained for 30 min was estimated as $9.2 \%$ at a nucleoside level, on the assumption that the molar absorptivity of the product is the same as that of CPrG.

The oxidative product was isolated and subjected to ESI-MS measurements. A molecular ionic peak of $\mathrm{m} / \mathrm{z} 705$ $\left([(2 M-2 \mathrm{H})+\mathrm{H}]^{+}, M\right.$ being the molecular weight of $\left.\mathrm{CPrG}\right)$ was 

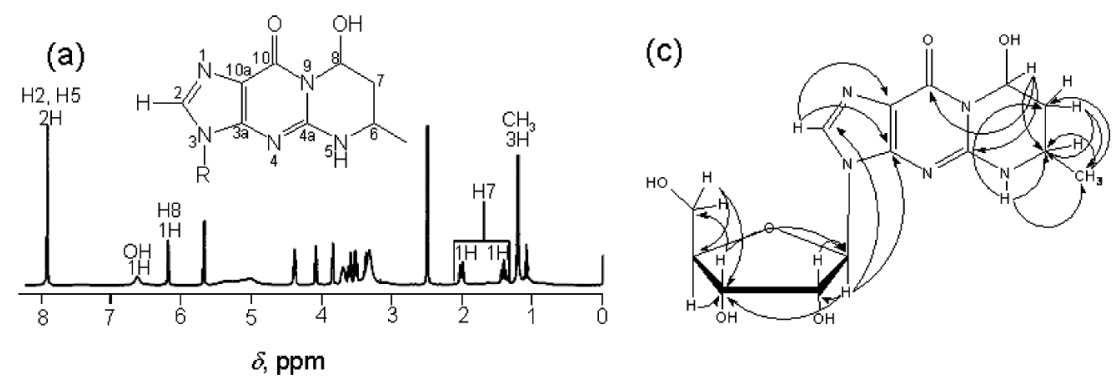

(b)

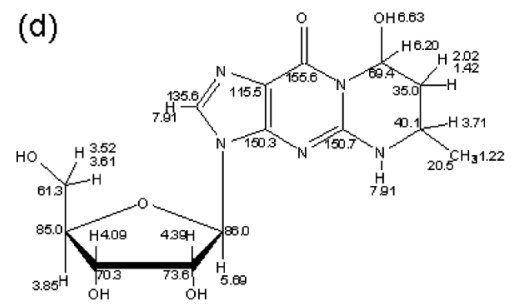

$\delta$, ppm

Fig. $4 \quad{ }^{1} \mathrm{H}-\mathrm{NMR}$ (a) and ${ }^{13} \mathrm{C}-\mathrm{NMR}$ spectra (b) of CPrG in DMSO- $d_{6}$, and the HMBC correlation (c) and the values (ppm) of chemical shift (d).

observed with a fragment peak of $\mathrm{m} / \mathrm{z} 573$ ([(2M-2Hribose) $+\mathrm{H}]^{+}$), which is expected to generate $\mathrm{D}-\mathrm{CPrG}$, as shown in Fig. 3(a). The dimer formation is experimentally deduced from MS/MS spectra showing a fragment peak of $\mathrm{m} / \mathrm{z} 441$, corresponding to the dimer of the cyclic propano adduct of guanine resulting in the elimination of riboses, as shown in Fig. 3(b). High-resolution ESI-MS results $(\mathrm{m} / \mathrm{z}=727.24117$, $\left.[(2 \mathrm{M}-2 \mathrm{H})+\mathrm{Na}]^{+}\right)$provide the chemical formula for the oxidative product as $\mathrm{C}_{28} \mathrm{H}_{36} \mathrm{~N}_{10} \mathrm{O}_{12}$. The MS results strongly suggest that electrooxidation of $\mathrm{CPrG}$ derives a dimeric compound of $\mathrm{CPrG}$.

\section{NMR spectra}

Figure 4 shows the ${ }^{1} \mathrm{H}-\mathrm{NMR}$ and ${ }^{13} \mathrm{C}-\mathrm{NMR}$ spectra of $\mathrm{CPrG}$ and the correlations obtained from heteronuclear multiple bond connectivity (HMBC) spectra. The $3 \mathrm{H}$-signal at $1.22 \mathrm{ppm}$ and the two $1 \mathrm{H}$-signals at 1.42 and $2.02 \mathrm{ppm}$ in the ${ }^{1} \mathrm{H}-\mathrm{NMR}$ spectrum were easily assigned to methyl protons at the C6 position and C7-methylene protons (refer to the structure in Fig. 4(a) for numbering of the atoms), respectively. Addition of $\mathrm{D}_{2} \mathrm{O}$ to the sample solution eliminates the 6.63-ppm signal and changes the integrated intensity $(2 \mathrm{H})$ of the 7.91-ppm signal to $1 \mathrm{H}$. On the other hand, the 7.91-ppm signal ( $\left.{ }^{1} \mathrm{H}-\mathrm{NMR}\right)$ correlates with the methyl carbon and the $3 \mathrm{a}$ and $10 \mathrm{a}$ carbons $\left({ }^{13} \mathrm{C}-\mathrm{NMR}\right)$ on the HMBC spectra, as shown in Fig. 4(c). Therefore the $2 \mathrm{H}$-signal at $7.91 \mathrm{ppm}$ and the $1 \mathrm{H}$-signal at $6.63 \mathrm{ppm}$ are reasonably assigned to overlapping of the $\mathrm{H} 2$ and $\mathrm{H} 5$ protons, and to the $\mathrm{OH}$ proton, respectively. Detailed analyses of the HMBC correlations give the assignments of the ${ }^{1} \mathrm{H}-\mathrm{NMR}$ and ${ }^{13} \mathrm{C}-\mathrm{NMR}$ signals shown in Fig. 4(d). The assignment of the ${ }^{1} \mathrm{H}-\mathrm{NMR}$ spectra of $\mathrm{CPrG}$ completely corresponds with the data previously reported. ${ }^{22}$

Figure 5 shows the ${ }^{1} \mathrm{H}-\mathrm{NMR}$ spectra of D-CPrG. Two resonances for the equivalent protons in two CPrG-monomers linked with each other are detected, except for the 8-ppm signals assigned to $\mathrm{H} 2$ and $\mathrm{H} 5$ in CPrG-monomer. The usual case is given by the methyl protons observed at 0.99 and $1.27 \mathrm{ppm}$ in the dimer. This means that $\mathrm{D}-\mathrm{CPrG}$ does not have the symmetric structure linked by the homo-coupling. The dimer cross-linked at $\mathrm{C} 2$ and $\mathrm{N} 5$ is deduced from the $1 \mathrm{H}$-signals at 7.45 and

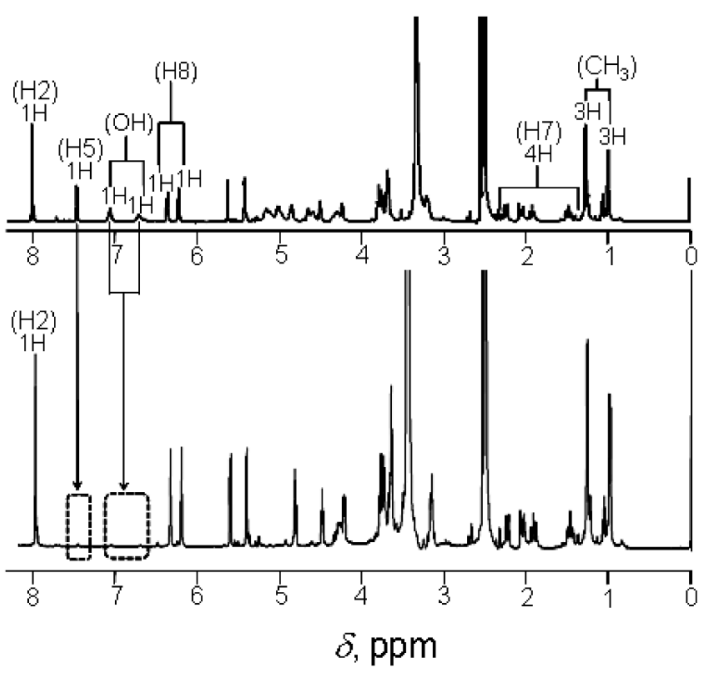

Fig. 5 The ${ }^{1} \mathrm{H}-\mathrm{NMR}$ spectra of D-CPrG in DMSO- $d_{6}$ (upper) and in DMSO- $d_{6}$ containing $\mathrm{D}_{2} \mathrm{O}$ (lower).

$8.00 \mathrm{ppm}$ observed for the dimer, assigned to $\mathrm{H} 5$ and $\mathrm{H} 2$, respectively, and from the effect of $\mathrm{D}_{2} \mathrm{O}$ addition shown in Fig. 5 (lower). Figure 6 shows the double quantum filtered-correlation spectrum (DQF-COSY) of D-CPrG. The correlations were observed between $\mathrm{H} 8$ and $\mathrm{OH}$ protons in the two monomers, indicating that the $6.70-$ and $7.05-\mathrm{ppm}$ signals in the ${ }^{1} \mathrm{H}-\mathrm{NMR}$ spectrum correspond to the $\mathrm{OH}$ protons. Therefore the 7.45- and 8.00-ppm signals are assigned to the $\mathrm{H} 5$ and $\mathrm{H} 2$ protons, respectively, and a set of $\mathrm{H} 2$ and $\mathrm{H} 5$ signals are eliminated in D-CPrG. A cross-linkage between the 2 and 5 positions in D-CPrG is rationally deduced from the NMR spectral features.

Unambiguous evidence for the C2-N5 linking in the dimer was obtained from the ${ }^{13} \mathrm{C}$-NMR and DEPT135 spectra, as shown in Fig. 7. Similar resonances for the equivalent carbons in two CPrG-monomers linked with each other are detected in 


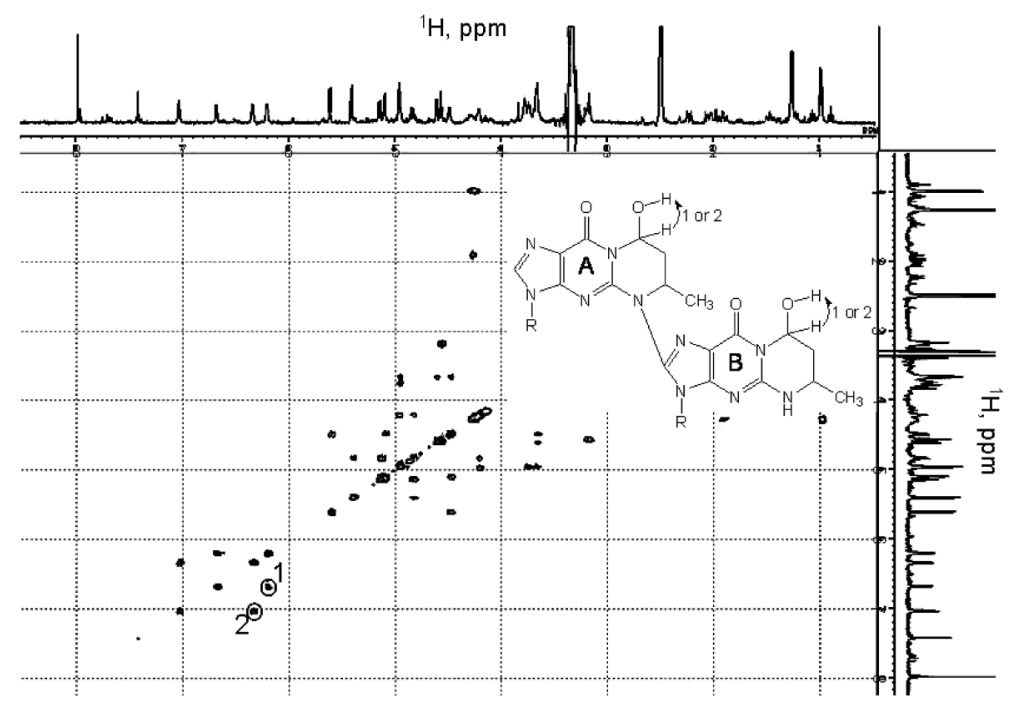

Fig. 6 DQF-COSY spectrum of D-CPrG.

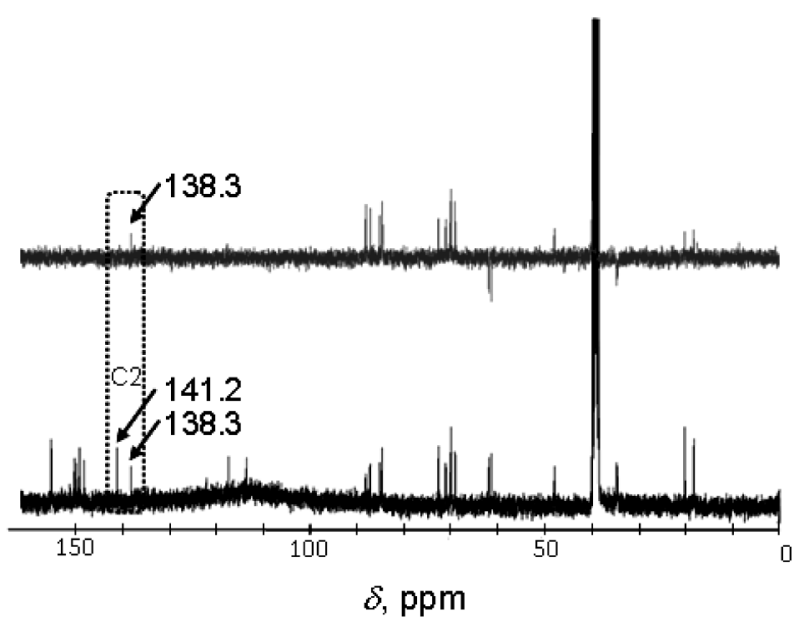

Fig. $7 \quad{ }^{13} \mathrm{C}-\mathrm{NMR}$ (lower) and DEPT135 spectra (upper) of D-CPrG in DMSO- $d_{6}$.

the ${ }^{13} \mathrm{C}$-NMR spectrum of D-CPrG as well as for the equivalent protons in the ${ }^{1} \mathrm{H}-\mathrm{NMR}$ spectrum. In addition, the presence of the quaternary $\mathrm{C} 2$ carbon in the dimer has been inferred from the elimination of the 141.2-ppm signal assigned to one of the $\mathrm{C} 2$ carbons in the DEPT135 spectrum. Further evidence for the cross-linking at $\mathrm{C} 2$ carbon is given by the 2-dimensional HMBC spectrum of D-CPrG, as shown in Fig. 8. The correlation involved by $\mathrm{H} 2$ hydrogen, which is denoted by 7 in Fig. 8, is observed for only one of the CPrG moieties in D-CPrG, indicating that $\mathrm{H} 2$ hydrogen does not exist in the other $\mathrm{CPrG}$ moiety. The correlations denoted by 2 and 5 in Fig. 8 also supported the assignment of the 138.3- and 141.2-ppm signals to the $\mathrm{C} 2$ carbons in the ${ }^{13} \mathrm{C}-\mathrm{NMR}$ spectrum.

Detailed analyses of the DQF-COSY, DEPT135 and HMBC spectra, and comparison with assignment of the NMR spectra for the $\mathrm{CPrG}$ monomer give the assignment of the ${ }^{1} \mathrm{H}-\mathrm{NMR}$ and ${ }^{13} \mathrm{C}-\mathrm{NMR}$ signals of $\mathrm{D}-\mathrm{CPrG}$ as follws $(\delta / \mathrm{ppm}) .{ }^{1} \mathrm{H}-\mathrm{NMR}$ in DMSO- $d_{6}$ (Fig. 5): $8.00(1 \mathrm{H}, \mathrm{s}, \mathrm{H}-2 \mathrm{~A}), 7.45$ (1H, br s, H-5B), $7.05(1 \mathrm{H}$, br s, $8-\mathrm{OH}), 6.70(1 \mathrm{H}$, br s, $8-\mathrm{OH}), 6.35(1 \mathrm{H}, \mathrm{s}, \mathrm{H}-8)$, $6.21(1 \mathrm{H}, \mathrm{s}, \mathrm{H}-8), 5.62\left(1 \mathrm{H}, \mathrm{d}, J=5.5 \mathrm{~Hz}, \mathrm{H}-1^{\prime} \mathrm{A}\right), 5.41(1 \mathrm{H}, \mathrm{d}$,
$\left.J=5.9 \mathrm{~Hz}, \mathrm{H}-1^{\prime} \mathrm{B}\right), 4.84\left(1 \mathrm{H}, \mathrm{s}, \mathrm{H}-2^{\prime} \mathrm{B}\right), 4.49$ (1H, m, H-2'A), $4.28(1 \mathrm{H}, \mathrm{m}, \mathrm{H}-6), 4.22\left(1 \mathrm{H}, \mathrm{s}, \mathrm{H}-3^{\prime} \mathrm{B}\right), 3.76\left(4 \mathrm{H}, \mathrm{m}, \mathrm{H}-4^{\prime} \mathrm{B}\right.$, H-5'B, H-6), 3.66 (3H, m, H-3'A, H-5'A), 3.20 (1H, s, H-4'A), $2.23(1 \mathrm{H}$, br d, $J=13.9 \mathrm{~Hz}, \mathrm{H}-7), 2.04(1 \mathrm{H}$, br d, $J=13.9 \mathrm{~Hz}$, H-7), 1.92 (1H, m, H-7), 1.47 (1H, m, H-7), 1.27 (3H, d, $J=$ $\left.6.2 \mathrm{~Hz}, 6-\mathrm{CH}_{3}\right), 0.99\left(3 \mathrm{H}, \mathrm{d}, J=6.4 \mathrm{~Hz}, 6-\mathrm{CH}_{3}\right) .{ }^{13} \mathrm{C}-\mathrm{NMR}$ in DMSO- $d_{6}$ (Fig. 7): 155.3 (q, C-10), 155.2 (q, C-10), 150.3 (q, C-4a), 149.9 (q, C-4a), 149.2 (q, C-3aB), 148.2 (q, C-3aA), 141.2 (q, C-2B), 138.3 (t, C-2A), 117.6 (q, C-10a), 113.8 (q, C-10a), 88.4 (t, C-1'), 87.5 (t, C-1'), 85.4 (t, C-4'), 84.9 (t, C-4'), 73.0 (t, C-2'A), 71.4 (t, C-2'B), 70.5 (t, C-3'), 70.3 (t, C-3'), 69.5 (t, C-8), 69.3 (t, C-8), $62.2\left(\mathrm{~d}, \mathrm{C}-5^{\prime}\right), 61.6\left(\mathrm{~d}, \mathrm{C}-5^{\prime}\right), 48.3(\mathrm{t}$, C-6), 40.4 (t, C-6), 35.1 (d, C-7), 34.9 (d, C-7), 20.6 (s, 6-CH $\mathrm{CH}_{3}$, $18.7\left(\mathrm{~s}, 6-\mathrm{CH}_{3}\right)$.

\section{$U V$ spectra of $D-C P r G$}

The UV spectrum of D-CPrG reflects the conjugated property of the CPrG-monomers. Red-shifts of the absorption maxima and increases in the intensities have not been observed for the spectrum of D-CPrG compared to that of CPrG, as shown in Fig. 9. This means that D-CPrG preferentially adopts the twisted conformation between two monomers due to steric crowding. In fact, the B3LYP/6-31+G(d,p) geometry of D-CPrG linked by $\mathrm{C} 2$ and $\mathrm{N} 5$ positions of monomers is optimized in the structure with a dihedral angle of $85^{\circ}$ for two monomers. $\mathrm{CNDO} / \mathrm{S}-\mathrm{CI}$ calculations were performed to gain more insight into the relation between the spectra and the conformation of the dimer. Table 1 lists the experimental and calculated data on the $\pi-\pi^{*}$ transitions of CPrG and D-CPrG in a ultra-violet region. The calculation results gave the absorption bands in longer wavelength than the experimental bands, but the relative spectral features of CPrG as well as D-CPrG are well simulated by the calculation results. On the other hand, the dimer obtained by the homo-coupling at $\mathrm{C} 2$ positions was optimized in the nearly planar structure with a dihedral angle of $31^{\circ}$. The CNDO/S-CI calculation results for the structure gave the intensified absorption peak at $355 \mathrm{~nm}$ with an oscillator strength of 0.93 due to the conjugation of two monomers. Such results strongly suggest that D-CPrG is conformed by the C2-N5 cross-linking. 


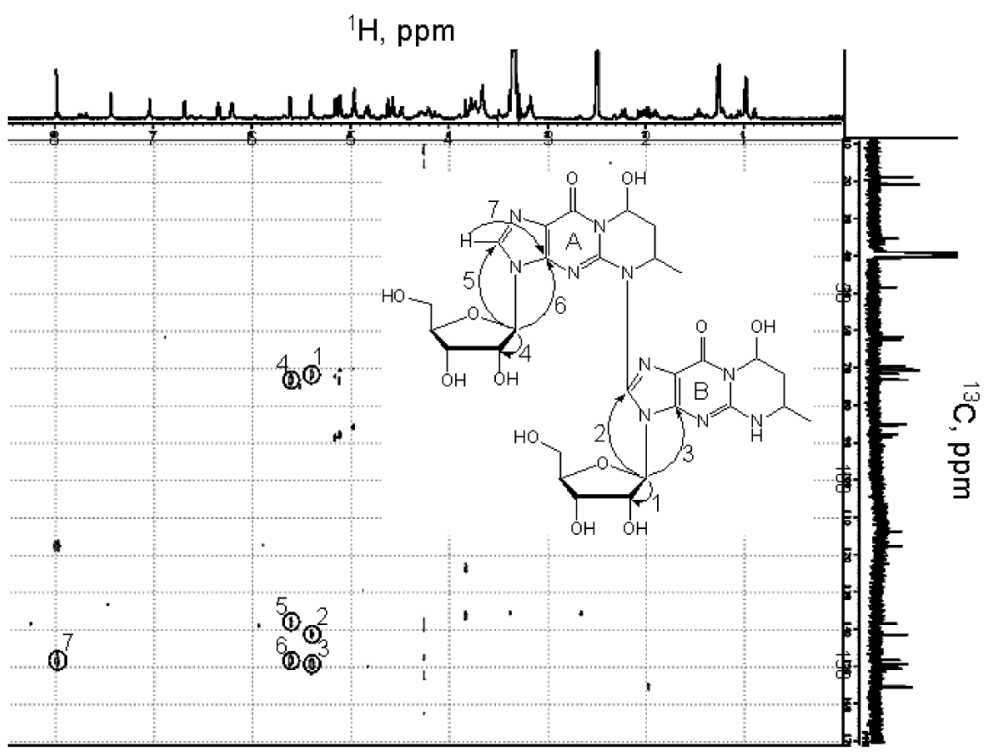

Fig. 8 HMBC spectrum and HMBC correlations of D-CPrG.

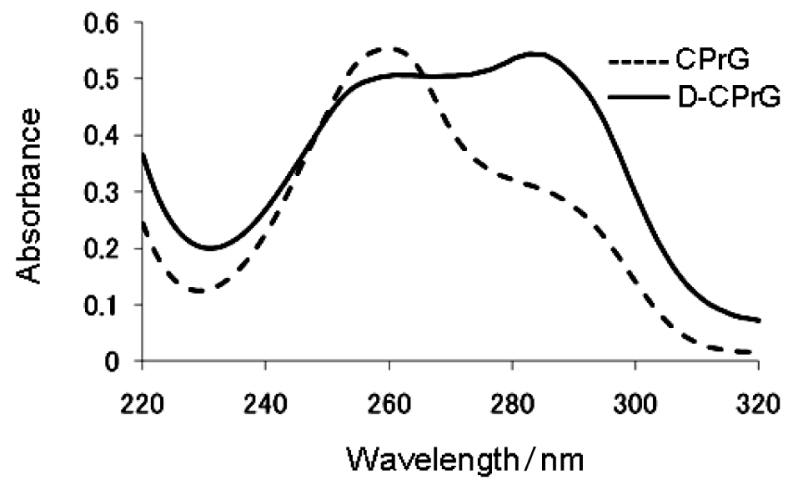

Fig. 9 UV spectra of $4.0 \times 10^{-5} \mathrm{~mol} \mathrm{dm}^{-3} \mathrm{CPrG}$ and $4.1 \times 10^{-5}$ $\mathrm{mol} \mathrm{dm}^{-3} \mathrm{D}-\mathrm{CPrG}$ in water.

Table 1 Spectral data and CNDO/S-CI calculation results for the absorption bands of $\mathrm{CPrG}$ and $\mathrm{D}-\mathrm{CPrG}$ in an ultraviolet region

\begin{tabular}{|c|c|c|c|c|c|}
\hline \multirow[b]{2}{*}{ Compound } & \multicolumn{2}{|c|}{ Observed $^{\mathrm{a}}$} & \multicolumn{3}{|r|}{ Calculated ${ }^{b}$} \\
\hline & $\begin{array}{c}\lambda_{\max } / \\
\mathrm{nm}\end{array}$ & $\varepsilon_{\max }$ & $\begin{array}{c}\lambda_{\text {calcd }} / \\
\mathrm{nm}\end{array}$ & $f_{\text {calcd }}$ & $\begin{array}{l}\text { Main configuration, } \\
\%^{c}\end{array}$ \\
\hline \multirow[t]{2}{*}{ CPrG } & $290(\mathrm{sh})^{\mathrm{d}}$ & 7000 & 350.0 & 0.250 & $92(45 / 46)$ \\
\hline & 260 & 14000 & 290.9 & 0.356 & $87(45 / 47)$ \\
\hline \multirow[t]{4}{*}{$\mathrm{D}-\mathrm{CPrG}$} & 283 & 13000 & 332.1 & 0.433 & $66(89 / 90), 22(88 / 91)$ \\
\hline & & & 324.1 & 0.104 & $\begin{array}{l}24(88 / 91), 19(86 / 93), \\
11(89 / 90), 7 \text { (81/93), } \\
6(86 / 91)\end{array}$ \\
\hline & $255(\mathrm{sh})^{\mathrm{d}}$ & 12600 & 280.1 & 0.313 & 78 (89/92), $6(88 / 93)$ \\
\hline & & & 272.1 & 0.456 & $\begin{array}{l}72(88 / 93), 8 \text { (89/93), } \\
7 \text { (88/92) }\end{array}$ \\
\hline
\end{tabular}

a. These are the values at the maximum intensity in water. b. The electronic transitions with oscillator strengths $\left(f_{\text {calcd }}\right)$ larger than 0.1 were listed.

c. For example, $(45 / 46)$ means a singly excited configuration from the 45 th occupied $\pi$-MO $\left(\psi_{45}\right)$ to the 46 th unoccupied $\pi$-MO $\left(\psi_{46}\right)$.

d. The letter in parentheses indicates a shoulder band.

e. The molecular geometry for the $\mathrm{C} 2-\mathrm{N} 5$ cross-linked structure is fully optimized by B3LYP/6-31+G(d,p) calculations.

\section{Conclusions}

CPrG is easily oxidized to form a cross-linked dimer (D-CPrG) as an oxidative product of CPrG. On the basis of product analyses with MS, NMR and UV spectra, we concluded that D-CPrG preferentially adopts the N5-C2 cross-linking. In double-stranded DNA, dimer formation strongly depends upon the base sequence. Simple molecular modeling suggested that a preference for intrastrand cross-linked dimer formation at the CPrG-CPrG sequence is favorable. The formation of D-CPrG as an oxidative lesion of the acetaldehyde-derived cyclic propano adduct of guanosine is anticipated to interfere with DNA replication and to contribute to acetaldehyde-mediated genotoxicity.

\section{References}

1. J. Cadet, T. Douki, and J.-L. Ravanat, "Damage to Nucleic Acids, Redox-Genome Interactions in Health and Disease," ed. J. Fuchs, M. Podda, and L. Packer, 2003, Chap. 8, Marcel Dekker, Inc., New York, 145.

2. V. Labet, C. Morell, A. Grand, J. Cadet, P. Cimino, and V. Barone, Org. Biomol. Chem., 2008, 6, 3300.

3. M. Sako, S. Inagaki, Y. Esaka, and Y. Deyashiki, Tetrahedron Lett., 2003, 44, 7303.

4. M. Sako, S. Inagaki, Y. Esaka, and Y. Deyashiki, Bioorg. Med. Chem. Lett., 2003, 13, 3497.

5. S. Inagaki, Y. Esaka, M. Goto, Y. Deyashiki, and M. Sako, Biol. Pharm. Bull., 2004, 27, 273.

6. S. Inagaki, Y. Esaka, Y. Deyashiki, M. Sako, and M. Goto, J. Chromatogr., A, 2003, 987, 341.

7. S. Zhang, P. W. Villalta, M. Wang, and S. S. Hecht, Chem. Res. Toxicol., 2006, 19, 1386.

8. Y. Lao and S. S. Hecht, Chem. Res. Toxicol., 2005, 18, 711.

9. S. Stein, Y. Lao, I. Y. Yang, S. S. Hecht, and M. Moriya, Mutat. Res., 2006, 608, 1.

10. H. Murakami, Y. Esaka, and B. Uno, Chem. Pharm. Bull., 2009, 57, 1434.

11. I. Saito, T. Nakamura, K. Nakatani, Y. Yoshioka, 
K. Yamaguchi, and H. Sugiyama, J. Am. Chem. Soc., 1998, 120, 12686.

12. K. Nakatani, C. Dohno, and I. Saito, J. Am. Chem. Soc., 2001, 123, 9681.

13. M. Sako and I. Yaekura, Tetrahedron, 2002, 58, 8413.

14. M. J. Frisch, G. W. Trucks, H. B. Schlegel, G. E. Scuseria, M. A. Robb, J. R. Cheeseman, J. A. Montgomery, Jr., T. Vreven, K. N. Kudin, J. C. Burant, J. M. Millam, S. S. Iyengar, J. Tomasi, V. Barone, B. Mennucci, M. Cossi, G. Scalmani, N. Rega, G. A. Petersson, H. Nakatsuji, M. Hada, M. Ehara, K. Toyota, R. Fukuda, J. Hasegawa, M. Ishida, T. Nakajima, Y. Honda, O. Kitao, H. Nakai, M. Klene, X. Li, J. E. Knox, H. P. Hratchian, J. B. Cross, V. Bakken, C. Adamo, J. Jaramillo, R. Gomperts, R. E. Stratmann, O. Yazyev, A. J. Austin, R. Cammi, C. Pomelli, J. W. Ochterski, P. Y. Ayala, K. Morokuma, G. A. Voth, P. Salvador, J. J. Dannenberg, V. G. Zakrzewski, S. Dapprich, A. D. Daniels, M. C. Strain, O. Farkas, D. K. Malick, A. D. Rabuck, K. Raghavachari, J. B. Foresman, J. V. Ortiz, Q. Cui, A. G. Baboul, S. Clifford, J. Cioslowski, B. B. Stefanov, G. Liu,
A. Liashenko, P. Piskorz, I. Komaromi, R. L. Martin, D. J. Fox, T. Keith, M. A. Al-Laham, C. Y. Peng, A. Nanayakkara, M. Challacombe, P. M. W. Gill, B. Johnson, W. Chen, M. W. Wong, C. Gonzalez, and J. A. Pople, GAUSSIAN 03, 2004, Gaussian, Inc., Wallingford CT.

15. R. L. Ellis, G. Kuehnlenz, and H. H. Jaffé, Theor. Chim. Acta, 1972, 26, 131.

16. G. Kuehnlenz and H. H. Jaffé, J. Chem. Phys., 1973, 58, 2238.

17. B. Tinland, Mol. Phys., 1969, 16, 413.

18. N. O. Lipari and C. B. Duke, J. Chem. Phys., 1975, 63, 1748.

19. C. B. Duke and N. O. Lipara, J. Chem. Phys., 1975, 63, 1758.

20. P. Jacques, J. Faure, D. Achalvet, and H. H. Jaffé, J. Phys. Chem., 1981, 85, 473.

21. N. Mataga and K. Nishimoto, Z. Phys. Chem., N. F., 1957, 13,140 .

22. M. Wang, E. J. McIntee, G. Cheng, Y. Shi, P. W. Villalta, and S. S. Hecht, Chem. Res. Toxicol., 2000, 13, 1149. 